\title{
Stop the escalation before it begins by using the pediatric Behavior Response Team protocol
}

By Karen Adkins-Bley, RN, MSA, CPHRM, JD, Barbara K. Shaw, BA, LMSW, ACSW, Josh

Smith, Lead Security Officer, Eileen McMyler, MS, RN, BC
In today's world, clinicians need to be prepared to care for challenging patients and families that are struggling with the stress of illness and hospitalization and have inadequate coping skills. The University of Michigan Health System (UMHS) has developed a protocol identifying a team with representatives from psychiatry, security, and risk management to provide a rapid response in situations that historically have resulted in, at worst, sentinel/adverse events and at best, service disasters.

The pediatric BRT protocol formalizes the purpose of the team, how staff should access them, and the expectation for involved staff to debrief about the interventions at identified times. It has proven to be an effective intervention and allows clinicians to provide needed care to the patients.

\section{INTRODUCTION}

The hospital is inherently a stressful place for patients and their families. Add to that financial concerns, serious illness, and inadequate coping skills and you have all the necessary ingredients to impede an effective and therapeutic hospital stay. Patients and families are assessed on admission to the hospital when families, especially parents, are upset, overwhelmed, or scared. These families may demonstrate behaviors that make the staff and other patients uncomfortable, such as raised voices or agitated movements that seem physically aggressive and unreasonably demanding. Initially, caregivers will meet these reactions with empathy, validation, negotiation, reassurance, and extra attention. When these methods are not effective, the caregiver needs to identify additional strategies to deal with these behaviors. If, after consulting the unit social worker, staff are still unable to interact effectively with the patient and family, the clinicians should initiate the pediatric Behavior Response Team (BRT) protocol.

\section{PEDIATRIC BEHAVIOR RESPONSE TEAM PROTOCOL}

The protocol is designed to maintain a working environment that is safe, comfortable, and free from all types of threats, threatening behavior, and violence. The protocol articulates the responsibility of all staff, contractors, students, 
patients, and visitors of the expectation to maintain a climate that does not foster or tolerate acts of violence, threats, or aggression. The purpose of the protocol is to establish a health system guideline that responds to incidents of violence or threats of violence in the pediatric setting. ${ }^{1}$ The employee or care team is encouraged to resolve concerns with patients and families with the local social worker for that unit; however, any employee can request the pediatric BRT. Once it is decided that a BRT consult is necessary, the BRT members become involved with the concerned clinical team on the unit, the patient, and family.

\section{DEVELOPMENT OF THE PLAN}

The BRT is made up of 3 disciplines: a social worker, a security officer, and a risk manager consultant. These staff are available by pager $24 / 7$. When a call comes in to the pediatric BRT, 1 of the 3 disciplines will receive the initial page and debrief with the caller to decide whether to call the other 2 members and a pediatric psychiatrist or to schedule a meeting at a later time. At the agreedupon time, the BRT members will debrief and interview involved staff. The involved parents or visitors may be asked to leave the unit by unit leadership and/or security and will be given a time to return for a meeting. They are informed that their visiting privileges depend on their attendance at this meeting. The BRT team will facilitate a discussion with as many members of the treatment team as possible, formulating an appropriate plan based on the concerns that the staff have with the patient and family.

The social worker on the team will document the formulated plan, identifying the concerns, specific limitations, and restrictions being imposed, as well as the consequences of any violations. This plan is then sent via e-mail to the nurse manager, risk management, security, the attending physician, and the pediatric psychiatrist for review and comment. Changes are incorporated until there is unanimous approval. This process is on a fast track so that a finalized version can be formally disseminated to the involved person/groups (see Table 1).

\section{Table 1: Recipients of Agreed-Upon Family Plan}

1. Identified patient/family member/visitor

2. Nurse manager

3. Security

4. Medical records

5. Risk management

6. Attending physician

7. House manager

8. Bed manager
Table 2: Offices/Leadership Notified of Implementation of the Plan

1. The office of patient relations

2. Risk management

3. Chief executive officer

4. Pediatric hospital administrator

5. Office of clinical affairs

6. Administrator on call

7. Public relations

There is no requirement made for signatures, as it is not an agreement but a directive. Once the patient and family receive the plan, the BRT and medical team meet to discuss the approach in sharing the plan. It is crucial for all the team members to understand the importance of not engaging in a discussion to dispute the content of the plan or the imposed restrictions, only allowing clarification of the restrictions.

Immediately after the meeting and depending on the contents of the plan, the parents or visitors are escorted by security either back to the patient's room or out of the hospital. The identified patient/family/visitor names are sent to a number of key people (see Table 2).

In addition to this communication, a page is sent out to the administrator on call and the house manager, notifying them that a pediatric behavior plan has been put in place for the identified patient. The letter is entered into the online medical record. Staff in areas that might interact with the family are instructed to forward any calls related to the plan to the pediatric BRT social worker first and, if the social worker is unavailable, to the risk management consultant. Depending on the plan put into place and the compliance of the parents or visitors, follow-up meetings may be held to change restrictions at the discretion of unit leadership. The pediatric BRT social worker will round frequently to check in with the staff while guidelines are in effect.

\section{EVALUATION}

It is recommended to have two debriefing sessions scheduled with the staff from the unit where the incidents occurred to allow for maximum staff attendance. The pediatric behavioral team and the lead psychiatrist will meet twice a year to review the cases where plans were put in place and staff will be invited to attend to provide feedback on their cases. Since the formal adoption of this protocol in June of 2010, the BRT protocol has been implemented on average, once a month. Lessons learned involve gaps in communication to the appropriate administrators who were not prepared to take calls about the situations and handoffs to transferring units. 


\section{STRESSES THAT CHALLENGE COPING SKILLS}

It is helpful to recognize when family members are under the stress of a hospitalization, their coping skills may be impacted, leading to these disruptive and challenging behaviors requiring the need for more help.

It is important to recognize the behaviors necessitating the healthcare worker to use planned strategies. When an effective plan is not in place, the healthcare worker's initial response may be to feel trapped and helpless, then to withdraw, avoiding the relationship and finally, experience guilt. When a concern is raised, the clinical team should come together and discuss a unified approach without making excuses for patient and family behavior. It is important not to take the patient or family's behavior personally. In order for the clinician to be prepared, $s($ he) should perform reality checks with others, minimize exposure to triggers, minimize any visible reaction and realize that one cannot control what people think or do. Patients and family members who have less effective coping skills benefit from structure. Setting limits is designed to create that structure, bringing a sense of order, addressing boundary issues, and promoting the relationship. In addition to these benefits felt by the patient and family, they also create a sense of safety for the clinician.

There are communication techniques used to set limits more effectively, such as using "I" statements, staying focused on the message, and being aware of voice inflections and nonverbal communication behaviors. When the patient or family acts out, it is important for the clinician not to become defensive or to deny accusations. In addition, it is important not to counterattack or withdraw unless the situation becomes abusive. In an abusive situation, the clinician should set the limit and leave.

These patients and families are often angry, and it is important for the clinician to have strategies to cope with this rage. It is helpful to recognize the triggers that will escalate their behaviors as well as not to react to anger with more anger. Most importantly, it is critical for the clinician not to tolerate the behavior that is outside the limits that have been set.

In order to recognize patients and families that are at risk of escalating, it is helpful to assess their perceptions and behaviors. Are their actions based in reality? When these patients feel things are getting out of control they may react by using the defense mechanism of splitting, which identifies them in the right and the clinician as in the wrong. As such they lose memories of positive interactions with the clinician and can't see the clinician as someone who really wants to help provide the best care for them. When dealing with patients and families who are experiencing high levels of stress, be specific about expectations. Communicate one limit at a time, beginning with easier boundaries. Articulate consequences and always follow through. When these behaviors are being observed it is important to pull the clinical team members together, and if necessary contact the BRT to help develop and implement a plan. Recognize that the medical team may have to practice before they present the plan to the patient and family so that they are all saying the same thing. The clinical team has to agree not to disagree with each other in front of the patient or family. It is important to have a plan for communicating among the clinical team so that they regroup when the plan is not being followed or not working. Having a plan in place is not the same thing as accepting abuse and should not be confused as that, so if a clinician feels frightened, he or she should always request a backup and if the situation escalates, be prepared to call security.

\section{CONCLUSION}

In today's world, clinicians need to be prepared to care for these challenging patients and families, struggling with the stress of illness and hospitalization with inadequate coping skills. When empathy, validation, negotiation, and reassurance are incorporated into an individualized plan of care using available resources is ineffective, the BRT protocol is a useful and effective next step to allow clinicians to provide the care the patient is admitted to the hospital to receive.

\section{REFERENCE}

1. The Joint Commission. Preventing violence in the health care setting. http://www.jointcommission.org/ sentinel_event_alert_issue_45_preventing_violence _in_the_health_care_setting_/. Sentinel Event Alert, Issue 45. Published June 3, 2010. Accessed March 23, 2012.

\section{ABOUT THE AUTHORS}

Karen Adkins-Bley, RN, MSA, CPHRM, JD, is a risk management consultant in the department of risk management at the University of Michigan Health System located in Ann Arbor, Michigan. She has been in healthcare risk management for over 6 years at the University of Michigan Health Systems and works primarily with pediatric, obstetric, and gynecology services and related concerns and claims. She is a nurse-attorney with over 25 years of nursing experience and currently the Education Committee co-chair for the Michigan Society of Healthcare Risk Management involved in planning two educational days and presentations for the 2-day annual meeting. She has also worked with the University of Michigan Health Systems' nursing education department in obtaining nursing continuing education units for educational offerings for 3 years. Barbara $K$ Shaw BA, LMSW, ACSW, is a psychiatric social worker at the University of Michigan Medical Center in Ann Arbor Michigan with over 35 years in social work, working with children and adolescents with behavior and psychiatric issues. 
She has been the lead member of the Pediatric Behavior Response Team for over 3 years providing crisis intervention within the pediatric medical center, and responding to out of control and potentially dangerous situations involving patients and their families. She frequently presents lectures for the Graduate School of Social Work at the University of Michigan and also provides supervision and training for child psychiatry residents on family therapy. Josh Smith is a security officer at University of Michigan Medical Center in Ann Arbor Michigan. He has 14 years of experience as public safety officer with experience in dealing with violent and difficult people, responding to crisis events, and deescalating situations. He has extensive training and certification for public safety including healthcare security and safety and crisis intervention. He is also the lead officer for the Mott Children's Hospital at the University of Michigan Health System as well as a key member of the pediatric Behavior Response Team. Eileen McMyler, MS, RN, BC, is a project manager in the department of risk management at the University of Michigan Health System located in Ann Arbor, Michigan. She has been in healthcare risk management for the last 4 years and comes to the position with over 25 years of nursing experience. She is board certified in nursing continuing education, and her most recent clinical roles were in staff support as a clinical nurse specialist and nursing education specialist. 